\title{
Optical design and simulation of a new coherence beamline at NSLS-II
}

Garth J. Williams, Oleg Chubar, Lonny Berman, Yong S. Chu, Ian K. Robinson

Garth J. Williams, Oleg Chubar, Lonny Berman, Yong S. Chu, Ian K. Robinson, "Optical design and simulation of a new coherence beamline at NSLS-II," Proc. SPIE 10388, Advances in Computational Methods for X-Ray Optics IV, 103880E (23 August 2017); doi: 10.1117/12.2274387

Event: SPIE Optical Engineering + Applications, 2017, San Diego, California, United States 


\title{
Optical design and simulation of a new coherence beamline at NSLS-II
}

\author{
Garth J. Williams ${ }^{a}$, Oleg Chubar ${ }^{a}$, Lonny Berman ${ }^{a}$, Yong S. Chu ${ }^{a}$, and Ian K. Robinson ${ }^{a}$ \\ ${ }^{a}$ Brookhaven National Laboratory, PO BOX 5000, UPTON, USA
}

\begin{abstract}
We will discuss the optical design for a proposed beamline at NSLS-II, a late-third generation storage ring source, designed to exploit the spatial coherence of the X-rays to extract high-resolution spatial information from ordered and disordered materials through Coherent Diffractive Imaging, executed in the Bragg- and forward-scattering geometries. This technique offers a powerful tool to image sub-10 nm spatial features and, within ordered materials, sub-Angstrom mapping of deformation fields. Driven by the opportunity to apply CDI to a wide range of samples, with sizes ranging from sub-micron to tens-of-microns, two optical designs have been proposed and simulated under a wide variety of optical configurations using the software package Synchrotron Radiation Workshop. The designs, their goals, and the results of the simulation, including NSLS-II ring and undulator source parameters, of the beamline performance as a function of its variable optical components is described.
\end{abstract}

Keywords: Coherent Imaging, Instrumentation, Coherent Scattering, Wave Propagation

\section{INTRODUCTION}

The rapid increase in spectral brightness of storage-ring-based x-ray sources has motivated the development of new methods for determining structure. One such method, coherent diffractive imaging (CDI), directly employs the highly coherent x-ray beams provided by such sources. Traditionally, CDI is accomplished by exposing a sample to a coherent illumination and measuring the continuous far-field diffraction pattern that results from this interaction. The method was initially demonstrated ${ }^{1}$ in a forward-scattering geometry with an absorbing sample. Shortly thereafter, it was extended to the continuous diffraction around a Bragg spot-so-called Bragg CDI (BCDI)-that results from illuminating a small crystal with such a beam ${ }^{2}$. In the original geometry, the method provides an imaging technique that is independent of the limitations of x-ray lenses and mirrors, which is an attractive prospect given the limitations-such as large focal spots, low efficiency, positional stability, or energy-dependent focal lengths-imposed on structural studies by these optics. In addition to these advantages, BCDI is sensitive to subtle distortions of the crystalline lattice within the sample and, thereby, provides a means by which to measure both structure and deformation.

Since the initial demonstration, BCDI experiments have provided valuable information in recovering 3D structure and mapping strain therein ${ }^{3 ; 4}$. Today, it can even be applied to transient phenomena, such as mapping acoustic waves ${ }^{5}$, at modern $\mathrm{x}$-ray sources. The methods have been applied to semiconductor materials, where strain engineering is an exciting direction for increasing performance from solid state devices ${ }^{6}$. In the study of complex oxides, BCDI has been used to map the polarization of ferroelectric domains in thin films ${ }^{7}$, potentially leading to the engineering of ferroelectric domain walls in these materials and thus improved devices. Individual defects within a battery cathode may be imaged and tracked over charge-discharge cycles, revealing their motion ${ }^{8}$. In some of these cases, samples are scanned through the x-ray beam, while, in others, time resolution of great importance in probing the phenomena, which precludes a scanning methodology. In these latter cases, it is highly desirable to be able to change the x-ray spot size in a "zoomable" manner to accommodate the crystalline grain of interest. The ability to accommodate this variable spot size is a feature of optimized instruments at third generation storage ring sources ${ }^{9}$.

The NSLS-II ${ }^{10}$ is the most recent North American storage ring x-ray facility. With an ultimate spectral brightness ${ }^{11}$ as high as $10^{21} \mathrm{ph} / \mathrm{sec} / 0.1 \% \mathrm{bw} / \mathrm{mm}^{2} / \mathrm{mrad}^{2}$, it is capable of delivering exceptionally high-brightness $\mathrm{x}$-ray beams and is a prime candidate for performing next generation (B)CDI experiments. In designing a new instrument to exploit the very high spectral brightness of the source to perform state-of-the-art imaging experiments, considerable thought must be given to the optical design. In the analysis that follows, we use SRW ${ }^{12}$ 
with the Sirepo ${ }^{13}$ front-end to simulate the performance of two optical designs that seek to achieve the goal of delivering x-ray spots that are high-flux, have variable degrees of coherence, and can be smoothly changed from 1 to 10 microns in diameter. All simulations are performed with $8 \mathrm{keV}$ quasi-monochromatic radiation emerging from an in vacuum undulator using the NSLS-II ring parameters ${ }^{11}$.

\section{MOTIVATION}

CDI methods rely on the ability to accurately calculate the amplitude and phase of the x-ray field, which is most easily achieved when the incident x-ray beam is fully coherent. In general, the recovery of structural and strain information is accomplished by numerically propagating candidate fields between the detection and sample-exit planes. This propagation is accompanied by the imposition of some known sample information in the sample-exit plane-e.g., its composition, distribution, or shape-and of the measured detection plane data in the far field. This cycle is repeated iteratively until a self-consistent solution is found ${ }^{14}$. The method is extraordinarily sensitive to deviations from the assumed beam conditions because it employs this iterative algorithm to solve an ill-posed inverse problem. In the current case, this implies the need to control the degree of coherence of the x-ray beam and the complex amplitude of the beam incident upon the sample.

In the first demonstrations of BCDI, the samples under investigation were single, isolated crystals of about 1 micron in size ${ }^{2 ; 15}$. As the technique has matured, the class of applicable samples has grown substantially. It is now possible to image large sections of an extended crystal ${ }^{16}$ and individual grains within a polycrystal ${ }^{17}$. In many cases, these advances are enabled by the ability to scan precisely in an unchanging x-ray illumination. This drives the need for a stable optical system and a smooth illumination. A high-brightness beam emerging from a traditional undulator will have long transverse coherence lengths, but it will also be capable of producing a high flux density of coherent photons, which enables time-resolved measurements to be performed. Such experiments ${ }^{5 ; 18}$ will not necessarily tolerate a scanning geometry and, therefore, a variable x-ray spot size is highly desirable. An x-ray spot of around 10 micron diameter will be suitable for a wide variety of materials systems, where the typical grain size is of this order.

In parallel to the enhancements to BCDI described above, a considerable amount of work has been performed toward the goal of loosening the strict requirements on the longitudinal and transverse coherence of the x-ray beam. It has been shown that both transverse ${ }^{19 ; 20}$ and longitudinal ${ }^{21}$ partial coherence can be accounted for in CDI. Such advances will ultimately allow extremely high time resolution, but require that the collateral effects of energy dispersive optics be carefully evaluated. For this reason, our initial concepts employ Kirkpatrick-Baez mirror pairs as the final optic.

\section{PRELIMINARY DESIGN}

\subsection{Original Concept}

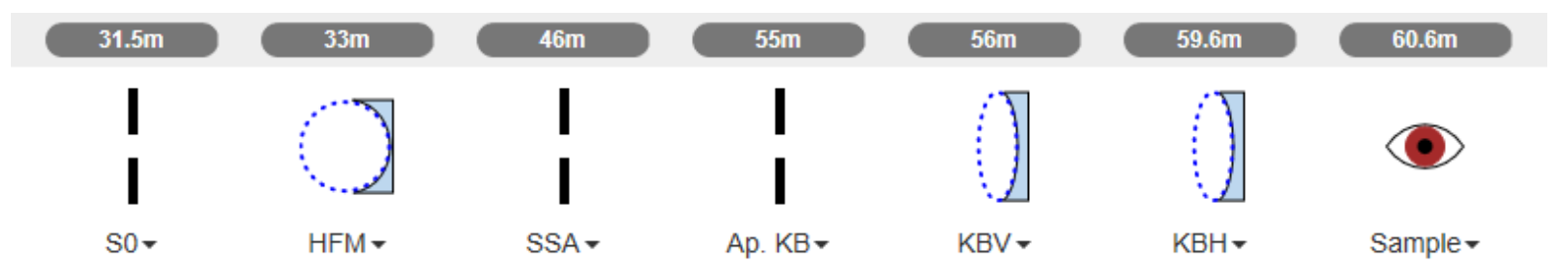

Figure 1. Original concept for the optical design of a Bragg coherent diffractive imaging beamline. The harmonic rejection/focusing mirror, HFM, is bendable and focuses the beam from the white-beam aperture, S0, at a secondary source plane. The secondary source aperture, SSA, forms the effective source for the final optics, a KB mirror pair comprised of $\mathrm{KBV}$ and KBH. The final spot size at the sample location is controlled by varying both apertures, S0 and SSA. Shown here is the graphical depiction of the concept from Sirepo/SRW ${ }^{13}$.

Our original concept for the optical system borrowed heavily from traditional x-ray microscopy beamline design: a white-beam aperture (S0), a bendable harmonic rejection mirror (HFM), followed by a double crystal monochromator (not shown), a smooth-bladed aperture in a secondary source plane (SSA), and final fixed-focus 


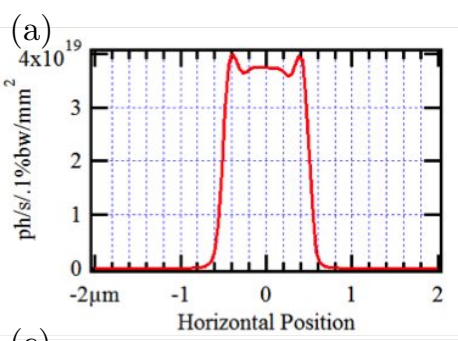

(c)

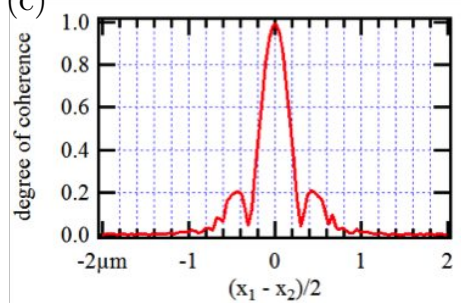

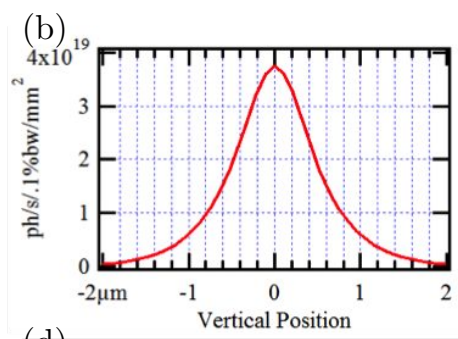

(d)

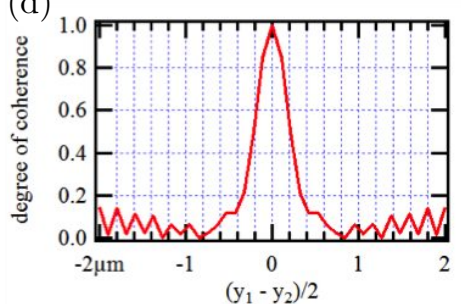

Figure 2. SRW-calculated profiles and degrees of coherence for the nominally 1 micron x-ray beam delivered by the original optical design. (a) and (b) are the calculated beam profile in the horizontal and vertical directions, while (c) and (d) are the estimated degrees of coherence for (a) and (b), optimized for maximizing flux while maintaining high coherence. The x-ray spot profile has an undesirable intensity variation in the horizontal, as seen in (a), and significant tails in the vertical, seen in (b). Calculations were performed for a photon energy of $8 \mathrm{keV}$.

optics(KBH and KBV) to re-image the secondary source at the sample location. Such a scheme is depicted graphically in Fig. 1. The advantage here is that the secondary source, given the fixed demagnification of the final optics, can be used to alter the final x-ray focal spot size. Distances shown are measured from the source point of the undulator.
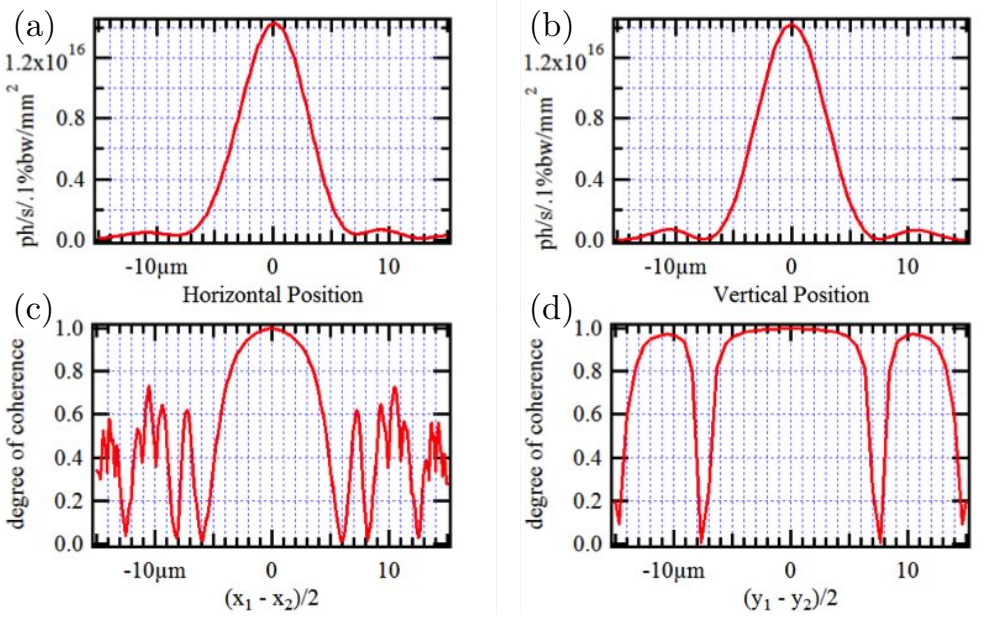

Figure 3. SRW-calculated profiles and degrees of coherence for the nominally 10 micron x-ray beam delivered by the original optical design. (a) and (b) are the calculated beam profile in the horizontal and vertical directions, while (c) and (d) are the estimated degrees of coherence for (a) and (b), optimized for maximizing flux while maintaining high coherence. While the intensity profiles of the focal spot in both the horizontal and vertical are smooth and sufficiently coherent, the flux delivered in this spot is estimated to be a factor of 6 lower when compared to Fig. 2. Calculations were performed for a photon energy of $8 \mathrm{keV}$.

In comparing the performance of the optical designs here and below, we will present, in addition to the calculated intensity profiles in the sample-plane, the degree of coherence of the final focal spot. We chose design parameters that will lead to the maximum possible flux, while allowing for a successful traditional CDI reconstruction to be performed. We define the spectral degree of coherence, following Mandel and Wolf ${ }^{22}$, Sec. 
Table 1. List of optical elements and their parameters for the simulation of the original concept. For the slits S0, SSA, and "Ap KB" we give horizontal and vertical gaps, for the mirror HFM we give the radius of curvature and the incident angle, for the $\mathrm{KB}$ focusing optics $\mathrm{KBV}$ and $\mathrm{KBH}$ we give the two radii for the elliptical figure.

\begin{tabular}{||l|c|c|c||}
\hline Optical Element & location $[\mathrm{m}]$ & small beam & large beam \\
\hline \hline source location & 0.000 & & \\
\hline S0 & 31.500 & 250 micron $(\mathrm{H})$ & 20 micron $(\mathrm{H})$ \\
& & $1 \mathrm{~mm}$ micron $(\mathrm{V})$ & 55 micron $(\mathrm{V})$ \\
\hline HFM & 33.000 & $\begin{array}{c}R_{x}=5329.21 \mathrm{~m} \\
\theta=3.5 \mathrm{mrad}\end{array}$ & $\begin{array}{c}R_{x}=5329.21 \mathrm{~m} \\
\theta=3.5 \mathrm{mrad}\end{array}$ \\
& & $15 \mathrm{micron}(\mathrm{H})$ & $1 \mathrm{~mm}(\mathrm{H})$ \\
SSA & 46.000 & $2 \mathrm{~mm}(\mathrm{~V})$ & $2 \mathrm{~mm}(\mathrm{~V})$ \\
\hline Ap KB & 55.000 & $1 \mathrm{~mm}(\mathrm{H})$ & $1 \mathrm{~mm}(\mathrm{H})$ \\
& & $1 \mathrm{~mm}(\mathrm{~V})$ & $2 \mathrm{~mm}(\mathrm{~V})$ \\
\hline KBV & 56.000 & $p=56 \mathrm{~m}$ & $p=56 \mathrm{~m}$ \\
& & $q=4.6 \mathrm{~m}$ & $q=4.6 \mathrm{~m}$ \\
\hline KBH & 59.600 & $p=59.6 \mathrm{~m}$ & $p=59.6 \mathrm{~m}$ \\
& & $q=1.0 \mathrm{~m}$ & $q=1.0 \mathrm{~m}$ \\
& & $\theta=3.5 \mathrm{mrad}$ & $\theta=3.5 \mathrm{mrad}$ \\
\hline sample & 60.600 & & \\
\hline
\end{tabular}

4.3.1, as:

$$
\mu\left(\boldsymbol{r}_{1}, \boldsymbol{r}_{2}, \omega\right)=\frac{W\left(\boldsymbol{r}_{1}, \boldsymbol{r}_{2}, \omega\right)}{\left[W\left(\boldsymbol{r}_{1}, \boldsymbol{r}_{1}, \omega\right)\right]^{1 / 2}\left[W\left(\boldsymbol{r}_{2}, \boldsymbol{r}_{2}, \omega\right)\right]^{1 / 2}}
$$

where $W\left(\boldsymbol{r}_{1}, \boldsymbol{r}_{2}, \omega\right)$ is the cross-spectral density. The full-width, half-maximum value of Eqn. (1) can be roughly interpreted as a "coherence length" when the function is smooth.

Figures 2 and 3 show SRW calculations of the horizontal and vertical focal distribution for two settings of the secondary source aperture one producing a micron-sized focal spot and the other producing a spot of about 7 microns.

It is clear that the intensity distribution of the 1-micron x-ray spot is highly structured in the horizontal, even when the beam possesses a moderate degree of partial coherence. This structure is highly undesirable for CDI-like experiments. As shown in the table 1, the scheme requires a very small SSA, the implementation of which would require careful engineering. Additionally, changing the focal spot size in this scheme causes large variations in the photon flux and the degree of coherence present in the beam delivered to the sample, with larger beams reducing flux by up to two orders of magnitude. We also note that the values provided in the table are not absolute, the degree of coherence could be larger while still matching the desired spot size. The "cost" for this, is to further reduce the flux incidence on the sample.

\subsection{Alternate Concept}

To address the shortcomings of the concept described in 3.1, we have developed a less traditional design. As shown in Fig. 4, the beamline now consists of: a white-beam aperture, (S0), a bendable harmonic rejection mirror (HFM), a vertical bendable mirror (VFM), a double crystal monochromator (not shown), a smoothbladed aperture at the entrance plane of the focusing optics (Ap KB), and final fixed-focus optics (KBH and KBV) to re-image the secondary source at the sample location. This concept is inspired by a traditional optical two-lens zoom design in both horizontal and vertical directions, re-imaging the coherence defining aperture S0.

Fig. 5 contains the line profiles and coherence estimates for the alternate concept. Here, the aperture S0 is used as a coherence defining aperture, i.e., its size is varied only to alter the degree of coherence of the final x-ray spot. The first two mirrors, HFM and VFM, create a virtual source of the x-ray beam. Not shown, a double crystal monochromator will follow these mirrors and is the only optical element between them and the 


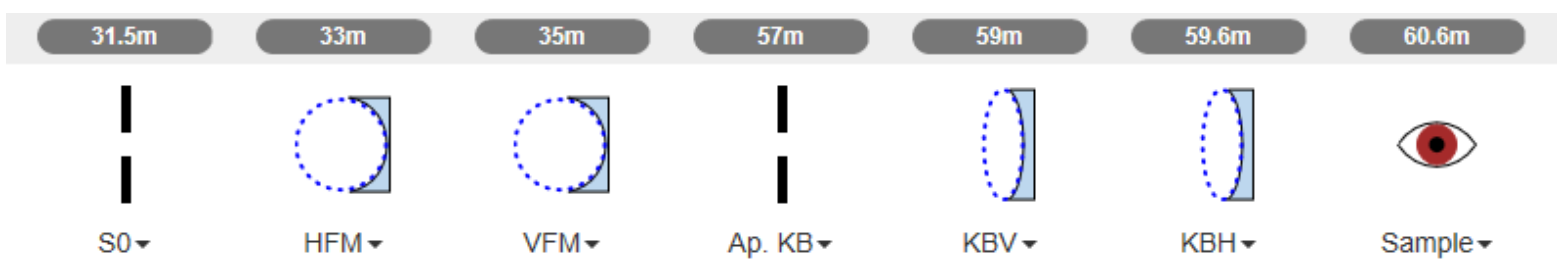

Figure 4. An alternate concept for the optical design of a Bragg coherent diffractive imaging beamline. The harmonic rejection and focusing mirror, HFM, and the vertical focusing mirror, VFM, are bendable and prepare the beam from the white-beam aperture, S0, for a fixed-figure KB mirror pair comprised of KBV and KBH. The final spot size at the sample location is controlled by the coordinating the bending of HFM and VFM with the displacements of KBV and $\mathrm{KBH}$. Shown here is the graphical depiction of the concept from Sirepo/SRW ${ }^{13}$.

aperture to the final focusing optics. Those final optics have been chosen to be fixed-figure KB mirrors. Mirrors are chosen for their achromatic focal length and the figure is fixed to ease the technical requirements in their manufacture, since it is highly desirable for these to be as close to wavefront-preserving as possible.
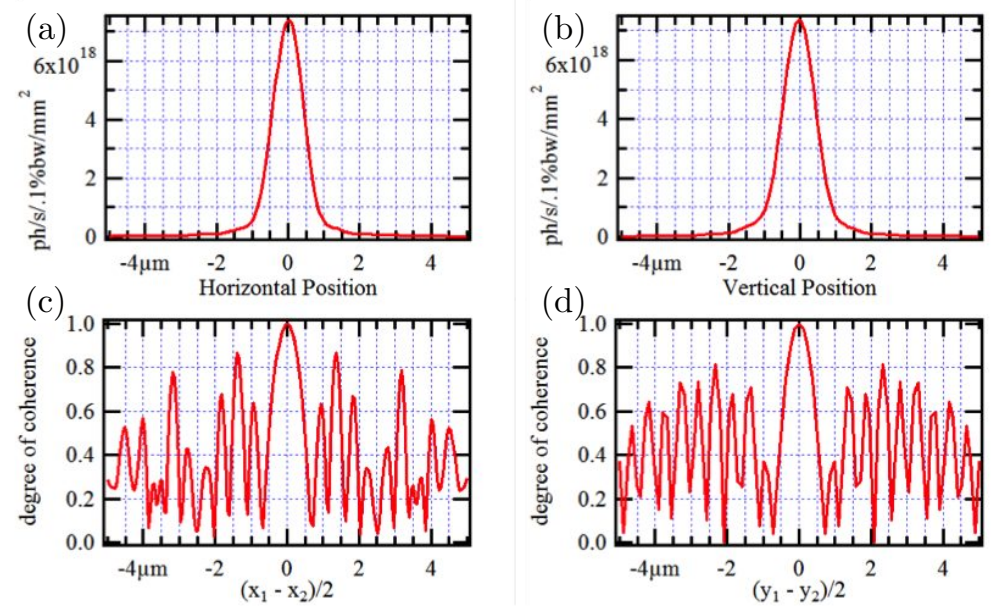

Figure 5. SRW-calculated profiles and degrees of coherence for the nominally 1 micron x-ray beam delivered by the original optical design. (a) and (b) are the calculated beam profile in the horizontal and vertical directions, while (c) and (d) are the estimated degrees of coherence for (a) and (b), optimized for maximizing flux while maintaining high coherence. The intensity profiles of the focal spot in both the horizontal and vertical are smooth and sufficiently coherent. Calculations were performed for a photon energy of $8 \mathrm{keV}$.

The focal spot size is changed by coordinating the bending of VFM and HFM with the displacement of KBV and KBH from the sample. As can be seen from panels (a) and (b) in Figs. 5 and 6, a smooth spot can be prepared over dimensions ranging from 1 to 10 microns. Additionally, the degree of coherence can be exactly controlled, by means of the size of the aperture S0, so that conditions ranging from full coherence to the natural coherence of the source can be delivered in those spot sizes.

Table 2 details the parameters needed to obtain the spot profiles in Figs. 5 and 6 . A principle feature of the design is that the size of the white-beam aperture is now decoupled from the final spot size, allowing a variable spot size with a variable degree of coherence. A significant design cost is incurred by the need to move the final fixed figure KB mirror pair significantly, with the vertical focusing mirror moving by about $1.5 \mathrm{~m}$. The variability of the spot size requires careful design of both the optics and the mechanical supports.

\section{FUTURE DIRECTIONS}

We have presented two very different optical schemes for providing a "zoomable" focus suitable for conducting state-of-the-art CDI experiments in either the forward scattering or Bragg geometry. The advantage of the first scheme is its robustness: the geometry is well-studied, has been implemented numerous times, and is resistant to 

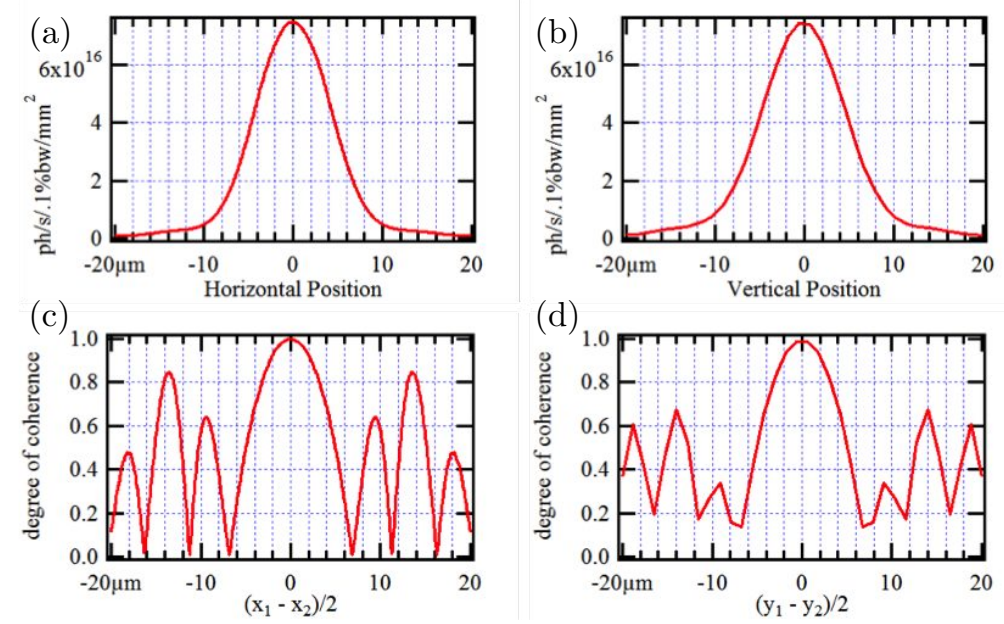

Figure 6. SRW-calculated profiles and degrees of coherence for the nominally 10 micron x-ray beam delivered by the original optical design. (a) and (b) are the calculated beam profile in the horizontal and vertical directions, while (c) and (d) are the estimated degrees of coherence for (a) and (b), optimized for maximizing flux while maintaining high coherence. The intensity profiles of the focal spot in both the horizontal and vertical are smooth and sufficiently coherent. The total flux delivered to the x-ray spot is within $10 \%$ of that delivered to the 1-micron spot. Calculations were performed for a photon energy of $8 \mathrm{keV}$.

Table 2. List of optical elements and their parameters for the simulation of the original concept.For the slits S0 and "Ap KB" we give horizontal and vertical gaps, for the mirrors HFM and VFM we give the radii of curvature and the incident angle, for the $\mathrm{KB}$ focusing optics $\mathrm{KBV}$ and $\mathrm{KBH}$ we give the two radii for the elliptical figure.

\begin{tabular}{|c|c|c|c|}
\hline Optical Element & location $[\mathrm{m}]$ & small beam & large beam \\
\hline source location & 0.000 & & \\
\hline S0 & 31.500 & $\begin{array}{l}35 \text { micron }(\mathrm{H}) \\
420 \text { micron }(\mathrm{V})\end{array}$ & $\begin{array}{l}35 \text { micron }(\mathrm{H}) \\
420 \text { micron }(\mathrm{V})\end{array}$ \\
\hline HFM & 33.000 & $\begin{aligned} R_{x} & =2408.91 \mathrm{~m} \\
\theta & =3.5 \mathrm{mrad}\end{aligned}$ & $\begin{array}{c}R_{x}=6625.0 \mathrm{~m} \\
\theta=3.5 \mathrm{mrad}\end{array}$ \\
\hline$\overline{V F M}$ & 35.000 & $\begin{array}{c}R_{x}=6086.71 \\
\theta=3.5 \mathrm{mrad}\end{array}$ & $\begin{aligned} R_{x} & =7181.07 \mathrm{~m} \\
\theta & =3.5 \mathrm{mrad}\end{aligned}$ \\
\hline Ap KB & 57.000 & $\begin{array}{l}1 \mathrm{~mm}(\mathrm{H}) \\
1 \mathrm{~mm}(\mathrm{~V})\end{array}$ & $\begin{array}{l}1 \mathrm{~mm}(\mathrm{H}) \\
2 \mathrm{~mm}(\mathrm{~V})\end{array}$ \\
\hline KBV & $\begin{array}{l}59.000 \text { (small) } \\
57.347 \text { (large) }\end{array}$ & $\begin{array}{c}p=8.6884 \mathrm{~m} \\
q=1.6 \mathrm{~m} \\
\theta=3.5 \mathrm{mrad}\end{array}$ & $\begin{array}{c}p=8.6884 \mathrm{~m} \\
q=1.6 \mathrm{~m} \\
\theta=3.5 \mathrm{mrad}\end{array}$ \\
\hline $\mathrm{KBH}$ & $\begin{array}{l}59.600 \text { (small) } \\
59.535 \text { (large) }\end{array}$ & $\begin{array}{c}p=21.767 \mathrm{~m} \\
q=1.0 \mathrm{~m} \\
\theta=3.5 \mathrm{mrad}\end{array}$ & $\begin{array}{c}p=21.767 \mathrm{~m} \\
q=1.0 \mathrm{~m} \\
\theta=3.5 \mathrm{mrad}\end{array}$ \\
\hline sample & 60.600 & & \\
\hline
\end{tabular}

vibrations and source fluctuations. However, in achieving the highest possible flux, it creates a variable coherence fraction of the final spot. Additionally, the SSA must be quite small to achieve the smaller spot size, which could lead to reliability issues.

The second scheme decouples the beam size from the coherent fraction in the final beam and provides a high-flux beam for all spot sizes. It is, unfortunately, more complex and therefore vulnerable to imperfections in optics and vibration coupled into the system through the experimental floor. More study is needed to accurately predict the real-world performance of this scheme. 
In the near future, we will conduct these simulation-based studies by:

- the addition of realistic mirror roughness and figure errors to the simulation;

- assessing the changes to the intensity and phase profile of the focused beam in the presence of instability by mis-steering beam through the simulated optical system;

- continuing to optimize mirror curvatures and ranges of motion to find realistic mirror parameters that meet the engineering constraints on the motion of the mirrors;

- the propagation distances between the front-end optics and the final focusing optics, as well as the focal lengths of the final mirrors; and

- considering the benefits and drawbacks of using energy-dispersive focusing optics to shape the beam.

\section{Acknowledgements}

This research used resources of the National Synchrotron Light Source II, a U.S. Department of Energy (DOE) Office of Science User Facility operated for the DOE Office of Science by Brookhaven National Laboratory under Contract No. DE-SC0012704. The work was paritally supported by the DOE SBIR grant DE-SC0011237.

\section{References}

[1] J. Miao, P. Charalambous, J. Kirz, and D. Sayre, "Extending the methodology of X-ray crystallography to allow imaging of micrometre-sized non-crystalline specimens," Nature 400(6742), p. 342, 1999.

[2] I. K. Robinson, I. A. Vartanyants, G. J. Williams, M. A. Pfeifer, and J. A. Pitney, "Reconstruction of the Shapes of Gold Nanocrystals Using Coherent X-Ray Diffraction," Physical Review Letters 87, Oct. 2001.

[3] M. A. Pfeifer, G. J. Williams, I. A. Vartanyants, R. Harder, and I. K. Robinson, "Three-dimensional mapping of a deformation field inside a nanocrystal," Nature 442, pp. 63-66, July 2006.

[4] M. C. Newton, S. J. Leake, R. Harder, and I. K. Robinson, "Three-dimensional imaging of strain in a single ZnO nanorod," Nature Materials 9, pp. 120-124, Feb. 2010.

[5] J. N. Clark, L. Beitra, G. Xiong, A. Higginbotham, D. M. Fritz, H. T. Lemke, D. Zhu, M. Chollet, G. J. Williams, M. Messerschmidt, B. Abbey, R. J. Harder, A. M. Korsunsky, J. S. Wark, and I. K. Robinson, "Ultrafast Three-Dimensional Imaging of Lattice Dynamics in Individual Gold Nanocrystals," Science 341, pp. 56-59, July 2013.

[6] M. Gailhanou, A. Loubens, J.-S. Micha, B. Charlet, A. A. Minkevich, R. Fortunier, and O. Thomas, "Strain field in silicon on insulator lines using high resolution x-ray diffraction," Applied Physics Letters 90, p. 111914, Mar. 2007.

[7] S. O. Hruszkewycz, M. J. Highland, M. V. Holt, D. Kim, C. M. Folkman, C. Thompson, A. Tripathi, G. B. Stephenson, S. Hong, and P. H. Fuoss, "Imaging Local Polarization in Ferroelectric Thin Films by Coherent X-Ray Bragg Projection Ptychography," Physical Review Letters 110, Apr. 2013.

[8] A. Ulvestad, A. Singer, J. N. Clark, H. M. Cho, J. W. Kim, R. Harder, J. Maser, Y. S. Meng, and O. G. Shpyrko, "Topological defect dynamics in operando battery nanoparticles," Science 348, p. 1344, June 2015.

[9] I. K. Robinson, "Optimisation of coherent X-ray diffraction imaging at ultrabright synchrotron sources," Zeitschrift für Kristallographie Supplements 2008, pp. 27-35, Feb. 2008.

[10] Brookhaven Science Associates LLC, "National Synchrotron Light Source II." https://www. bnl .gov/ps/.

[11] Brookhaven Science Associates LLC, "Summary of NSLS-II Source Properties." https://www.bnl.gov/ nsls2/project/source_properties.asp. 
[12] "Synchrotron Radiation Workshop (SRW)." https://github.com/ochubar/SRW.

[13] Radiasoft, "Sirepo: A framework for scientific cloud computing." https://github.com/radiasoft/sirepo.

[14] S. Marchesini, "Invited Article: A unified evaluation of iterative projection algorithms for phase retrieval," Review of Scientific Instruments 78, p. 011301, Jan. 2007.

[15] G. J. Williams, M. A. Pfeifer, I. A. Vartanyants, and I. K. Robinson, "Three-Dimensional Imaging of Microstructure in Au Nanocrystals," Physical Review Letters 90, Apr. 2003.

[16] S. O. Hruszkewycz, M. Allain, M. V. Holt, C. E. Murray, J. R. Holt, P. H. Fuoss, and V. Chamard, "High-resolution three-dimensional structural microscopy by single-angle Bragg ptychography," Nature Materials 16, pp. 244-251, Nov. 2016.

[17] A. Yau, W. Cha, M. W. Kanan, G. B. Stephenson, and A. Ulvestad, "Bragg coherent diffractive imaging of single-grain defect dynamics in polycrystalline films," Science 356(6339), pp. 739-742, 2017.

[18] J. N. Clark, J. Ihli, A. S. Schenk, Y.-Y. Kim, A. N. Kulak, J. M. Campbell, G. Nisbet, F. C. Meldrum, and I. K. Robinson, "Three-dimensional imaging of dislocation propagation during crystal growth and dissolution," Nature Materials 14, pp. 780-784, June 2015.

[19] G. J. Williams, H. M. Quiney, A. G. Peele, and K. A. Nugent, "Coherent diffractive imaging and partial coherence," Physical Review B 75, Mar. 2007.

[20] L. W. Whitehead, G. J. Williams, H. M. Quiney, D. J. Vine, R. A. Dilanian, S. Flewett, K. A. Nugent, A. G. Peele, E. Balaur, and I. McNulty, "Diffractive Imaging Using Partially Coherent X Rays," Physical Review Letters 103, Dec. 2009.

[21] B. Abbey, L. W. Whitehead, H. M. Quiney, D. J. Vine, G. A. Cadenazzi, C. A. Henderson, K. A. Nugent, E. Balaur, C. T. Putkunz, A. G. Peele, G. J. Williams, and I. McNulty, "Lensless imaging using broadband X-ray sources," Nat Photon 5, pp. 420-424, July 2011.

[22] L. Mandel and E. Wolf, Optical Coherence and Quantum Optics, Cambridge University Press, New York, 2008. 